\title{
B
}

Evidence Based Library and Information Practice

Article

\section{Developing a Comprehensive Search Strategy for Evidence Based Systematic Reviews}

Julia B. DeLuca

Information Specialist

Centers for Disease Control and Prevention

National Center for HIV/AIDS, Hepatitis, STD and TB Prevention

Division of HIV/AIDS Prevention

Prevention Research Branch

Atlanta, GA, United States of America

Email: zxz4@cdc.gov

Mary M. Mullins

Information Specialist

Centers for Disease Control and Prevention

National Center for HIV/AIDS, Hepatitis, STD and TB Prevention

Division of HIV/AIDS Prevention

Prevention Research Branch

Atlanta, GA, United States of America

Email: asu8@cdc.gov

Cynthia M. Lyles

Mathematical Statistician

Centers for Disease Control and Prevention

National Center for HIV/AIDS, Hepatitis, STD and TB Prevention

Division of HIV/AIDS Prevention

Prevention Research Branch

Atlanta, GA, United States of America

Email: $\underline{\text { cml6@cdc.gov }}$

Nicole Crepaz

Behavioral Scientist

Centers for Disease Control and Prevention

National Center for HIV/AIDS, Hepatitis, STD and TB Prevention

Division of HIV/AIDS Prevention

Prevention Research Branch

Atlanta, GA, United States of America

Email: ncc9@cdc.gov 


\author{
Linda Kay \\ Behavioral Scientist \\ Centers for Disease Control and Prevention \\ National Center for HIV/AIDS, Hepatitis, STD and TB Prevention \\ Division of HIV/AIDS Prevention \\ Prevention Research Branch \\ Atlanta, GA, United States of America \\ Email: 1sk0@cdc.gov \\ Sekhar Thadiparthi \\ Business Analyst \\ Centers for Disease Control and Prevention \\ National Center for HIV/AIDS, Hepatitis, STD and TB Prevention \\ Division of HIV/AIDS Prevention \\ Prevention Research Branch \\ Atlanta, GA, United States of America \\ Email: zkv9@cdc.gov
}

Received: 11 December 2007

Accepted: 10 February 2008

(C) 2008 De Luca et al. This is an open access article distributed under the terms of the Creative Commons Attribution License (http://Creativecommons.org/licenses/by/2.0), which permits unrestricted use, distribution, and reproduction in any medium, provided the original work is properly cited.

\begin{abstract}
Objective - Within the health care field it becomes ever more critical to conduct systematic reviews of the research literature to guide programmatic activities, policy-making decisions, and future research. Conducting systematic reviews requires a comprehensive search of behavioural, social, and policy research to identify relevant literature. As a result, the validity of the systematic review findings and recommendations is partly a function of the quality of the systematic search of the literature. Therefore, a carefully thought out and organized plan for developing and testing a comprehensive search strategy should be followed. This paper uses the HIV/AIDS prevention literature to provide a framework for developing, testing, and conducting a comprehensive search strategy looking beyond RCTs.
\end{abstract}

Methods - Comprehensive search strategies, including automated and manual search techniques, were developed, tested, and implemented to locate published and unpublished citations in order to build a database of HIV/AIDS and sexually transmitted diseases (STD) literature. The search incorporated various automated and manual search methods to decrease the chance of missing pertinent information. The automated search was implemented in MEDLINE, EMBASE, PsycINFO, Sociological Abstracts and AIDSLINE. These searches utilized both index 
terms as well as keywords including truncation, proximity, and phrases. The manual search method includes physically examining journals (hand searching), reference list checks, and researching key authors.

Results - Using automated and manual search components, the search strategy retrieved 17,493 articles about prevention of HIV/AIDS and STDs for the years 19882005. The automated search found $91 \%$, and the manual search contributed $9 \%$ of the articles reporting on HIV/AIDS or STD interventions with behavioural/biologic outcomes. Among the citations located with automated searches, $48 \%$ were found in only one database (20\% MEDLINE, $18 \%$ PsycINFO, 8\% EMBASE, $2 \%$ Sociological Abstracts).

Conclusions - Development of a comprehensive review of the literature requires searching multiple databases and methods of manual searching in order to locate all relevant citations. Understanding the project needs, recognizing the limitations and strengths of specific electronic databases, and being aware of other methods for developing and refining a search are vital in planning an effective and comprehensive search strategy. Reporting standards for literature searches as part of the broader push for procedurally transparent and reproducible systematic reviews is not only advisable, but good evidence based practice.

\section{Introduction}

Within evidence based health care it becomes ever more critical to conduct systematic reviews of research literature to guide programmatic activities, policymaking, and future research. This increasing reliance of the health care field (e.g., medicine, nursing, radiology, and social work) on evidence based research highlights the importance of a comprehensive and systematic search and examination of the best scientific literature (Perry 3). Research synthesis, including systematic reviews and meta-analysis, is a valuable tool in tracking down the strongest scientific evidence to move prevention into program practice and to answer clinical questions (Sackett 72).

High quality research synthesis consists of a systematic process for identifying, critically appraising, synthesizing, and translating the information. The first important step in systematic reviews is to develop and implement a search strategy for identifying the relevant scientific literature available (Jenuwine and Floyd 349; Howes et al. 101; Bruce and Mollison 14; McManus et al. 1562; Chalmers, Dickersin, and Chalmers 786; Crumley and Blackhall 167). Without identifying the relevant literature in a systematic, comprehensive, and reproducible way, the information retrieved in the search may not be complete and may potentially lead to differing and potentially conflicting recommendations (Conn et al. 181; Counsell 384). Despite the critical implications of a flawed or incomplete search, published systematic reviews and meta-analysis often provide a limited explanation of the search methods used to capture the literature (Booth 422; Weller 163; Zhang, Sampson, and MacGowan 5). Determining which electronic databases will be searched; knowing how to apply indexing terms, key words, and key phrases to refine a search; deciding whether both automated and manual components are necessary; and knowing how to combine various components are all important 
aspects of producing a high quality

systematic search.

The HIV/AIDS and STD prevention field has recently begun to rely on research synthesis to prioritize funding program efforts. The U.S. Center for Disease Control and Prevention's (CDC) Prevention Research Synthesis (PRS) Project has conducted many systematic reviews and meta-analyses to provide important recommendations for developing efficacious prevention programs. High risk groups, such as men who have sex with men (MSM), HIVpositive, African American, and Hispanic populations have been critically reviewed to evaluate the efficacy of behavioural interventions and to identify research gaps (Crepaz et al. "Prevention;" Crepaz et al. "Efficacy;" Herbst et al. "Meta-Analytic MSM;" Herbst et al. "Hispanic;" Herbst et al. "Effectiveness MSM;" Johnson et al.; Simoni et al.). Other types of systematic reviews have identified specific HIV behavioural interventions demonstrating strong evidence of efficacy in reducing HIV risk (Lyles et al. "Evidence-Based;" Evidence Based Interventions). The findings of these systematic reviews have promoted the US national dissemination of evidence based HIV intervention (Collins et al.; Neumann and Sogolow), and have been incorporated into the HIV prevention strategic plans of state health departments across the USA (Peterson and Randall; Shea et al.).

Systematic reviews of intervention research have typically focused on randomized controlled trials (RCTs) (Berry et al.; Boynton et al.; Crumley et al. "Resources"; Dickersin, Scherer, and Lefebvre; Helmer et al.; Higgins and Green; Jadad, Moher, and Klassen; Robinson and Dickersin; Savoie et al.; Watson and Richardson). In HIV prevention research, however, a wealth of intervention research exists that utilizes nonrandomized evaluation designs. Some recent articles have called for an expanded review of the scientific evidence by including these types of studies in order to provide a more integrated picture of the existing state-of-science and broader evidence based recommendations for public health practice (Atkins, Fink, and Slutsky; Victora, Habicht, and Bryce). Incorporating evidence from a broader range of literature beyond RCTs requires a more general systematic search of the literature and, thus, a more complex search strategy.

This paper is intended to provide a framework for developing, testing, and conducting a comprehensive search strategy looking beyond RCTs, using the PRS Project and its research into HIV/AIDS behavioural prevention literature between 1988 and 2005. The contributions of automated and manual searches, as well as the citations identified in each electronic database used in the automated search, were analyzed to assess their overall importance in information retrieval within the HIV/AIDS behavioural intervention research field.

\section{CDC's HIV/AIDS Prevention Research Synthesis Project}

An understanding of the context and purpose of a research synthesis project is essential to guide the development of the project's search strategy. The PRS Project was initiated in 1996 by the Division of HIV/AIDS Prevention (DHAP) at the CDC to translate the cumulative scientific literature into evidence based recommendations for guiding programmatic activities, policy-making, and future research. The specific goals of the project are to:

- $\quad$ systematically identify and catalog the HIV/AIDS or Sexually Transmitted Diseases (STD) behavioural prevention research literature; 
- conduct systematic reviews and meta-analyses to understand the overall efficacy of different types of behavioural interventions and to identify efficacious components of those interventions;

- identify behavioural interventions with evidence of efficacy; and

- assess gaps in the existing literature for future research (Sogolow et al.).

An internal database of HIV/AIDS behavioural prevention literature was created to serve as the resource for all PRS research synthesis activities. This database has been the foundation for many peerreviewed publications (Crepaz et al. "Prevention;" Crepaz et al. "Efficacy;" Crepaz et al. "HIV-Positive;" Passin et al.; Herbst et al. "Meta-Analytic MSM;" Herbst et al. "Hispanic;" Herbst et al. "Effectiveness MSM;" Lyles et al. "Best-Evidence").

Due to the varied goals of the PRS project, several unique requirements for establishing the PRS database had to be considered when developing a systematic search. The focus of the PRS project is to evaluate the efficacy of HIV behavioural interventions. Although this focus is relatively narrow, the goals of the PRS project required the inclusion of all HIV/AIDS and STD behavioural prevention research literature in the PRS database.

Thus, developing a broader scope in the systematic search allowed for reviews of background literature or formative research in HIV behavioural prevention. It was also important to understand that HIV prevention literature cuts across multiple research areas including medicine, public health, nursing, psychology, sociology, anthropology, and social work. Searching across a wide range of research fields is necessary to ensure the comprehensiveness of the PRS database. These features of the project and the literature made this an ideal topic area for illustrating a framework for developing a systematic search strategy that may be of use in a range of health and social care fields.

\section{Methods}

Developing a systematic search to accommodate the unique aspects of the PRS project required extensive planning and testing. To identify all relevant citations across multiple sources and within a broad scope, the search strategy consisted of automated and manual search components. The National Library of Medicine (NLM) estimates 13,000 to 14,000 biomedical journals are currently being published worldwide, and that these journals can be searched through various electronic databases (NLM Factsheet). No single electronic database offers complete indexing of all available citations; therefore multiple databases need to be searched (Crumley and Blackhall 167; Alpi 98). Manual searching was included to complement automated searches, because relying on electronic databases alone would have left the searches vulnerable to missing information or to errors in indexing.

\section{Automated Search}

Focusing the Topic Area

The focus of the PRS project - evaluating the efficacy of HIV behavioural interventions was separated into three unique and equally important domains to help structure and guide the development of the automated search:

- HIV, AIDS, or Sexually Transmitted Disease (STD)

- prevention, intervention, or evaluation

- behavioural or biologic outcomes measuring potential or actual HIV or STD risk reduction.

Citations referring to HIV/AIDS/STD behavioural prevention research were 
considered within PRS as "in-scope"

citations, whereas, citations that

encompassed all three domains (i.e., HIV/AIDS/STD behavioural intervention citations with behavioural or biologic evaluation data) were the primary focus of the PRS project.

\section{Database Selection}

The PRS project searched four electronic databases to cover a broad range of health related literature: MEDLINE, EMBASE, PsycINFO, and Sociological Abstracts using the Ovid platform. These are key databases for biomedical, psychological, behavioural science, and public health literature. AIDSLINE, a database specifically dedicated to HIV/AIDS research, was also included in the PRS search until its discontinuation in December 2000. The search was initially developed, tested, and implemented in MEDLINE, "the most widely used and studied database in health care" (Jadad, Moher, and Klassen 812). Core indexing terms, keywords, and key phrases were the main elements of the automated search component. Each step in developing the automated search component was conducted simultaneously for each of the three key domains.

Any group conducting systematic searching and review will ultimately realize there is a limited amount of time and resources available (Crumley and Blackhall 167; Jadad, Moher, and Klassen 812). Databases were selected according to the capabilities, time constraints, and resources available.

Databases considered and tested for inclusion, but ultimately not used were CINAHL, Current Contents, ERIC, and ISI Web of Knowledge (Social Science Citation Index and Science Citation Index Expanded). Results of those tests concluded that citations from CINAHL would have duplicated results and would not contribute significant findings beyond those identified in MEDLINE.
Current Contents was not selected due to the focus of the database - it did not offer specialized or significant insight into public health areas outside the findings of the other included databases. The focus of ERIC is on educational and youth populations, an area researched by another CDC entity, the Division of Adolescent and School Health (DASH). ISI's Web of Knowledge provides valuable linking information, but its indexing system does not use a controlled vocabulary. ISI databases were used in the manual search process to verify references between related articles.

\section{Testing Each Database}

Testing of each database consisted of using the same indexing, keywords, and phrases to retrieve and analyze the citations collected. A sample search strategy was created based on 75 HIV/AIDS or STD behavioural intervention evaluation citations. The search was used to determine if that group of articles would be retrieved by each database. This test sought to answer certain questions:

- How many citations were retrieved?

- Are these articles within the scope of the project?

- How many extraneous citations were collected with this search?

Testing was performed in all databases considered, but only the selected databases were fully searched.

\section{Developing an Automated Search}

The first step in developing the automated search component was to identify Medical Subject Headings (MeSH) terms in MEDLINE. The 75 previously mentioned evaluation citations were examined to create a list of MeSH terms by conducting inverse searching - identifying terms by analyzing the subject indexing in order to expand the search (Boynton et al. 141). Each potential MeSH term was evaluated in the National 
Library of Medicine's MeSH database, examining the entry term, scope note, subheadings, related terms, and previous indexing for each of the terms from the 75 citations. The list of MeSH terms was compiled separately for each of the three primary domains of the search. Initially, 116 possible MeSH terms were identified. After cross-checking the indexing of all 75 citations and then sampling citations retrieved with these terms, the pool narrowed to $42 \mathrm{MeSH}$ terms for the automated search component for MEDLINE.

Once completed for MEDLINE, the list of indexing terms was tested, and adapted where necessary, to accommodate differences in the remaining electronic databases. Matching subject terms between databases was complex in some cases. Sometimes similar indexing terms do not exist between databases, creating gaps in search components. The scope note for each indexing term contains both a definition and also lists of "used for" and "related terms" to help pinpoint the specific intent and usage of that subject heading. Each term was checked individually and then its related terms were also tested, making it possible to narrow or broaden the search as needed.

With indexing terms identified, the automated search was further refined by using subheadings (i.e., subcategories of descriptors within indexing terms). Subheadings, available only in MEDLINE, AIDSLINE and EMBASE, were used to refine an indexing term when the concept was too broad to be an effective search term (Greenhalgh 181). After carefully reviewing the scope notes, the "prevention and control" subheading was selected to increase the precision of the search. Adding this subheading effectively reduced the number of irrelevant citations and substantially decreased the volume of citations.

\section{Refining the Automated Search}

Another method for refining searches was to use the "explode" and "focus" options provided by the OVID search platform. For the HIV/AIDS/STD domain, using the focus option (indicated by an asterisk in OVID) ensured the search would return citations specific to a particular subject heading or subheading (e.g., "HIV infection/pc*”). For the intervention evaluation and outcome domains, focusing seemed to limit the automated search too much, while exploding ("EXP" in OVID) captured a large number of irrelevant citations. Using a variety of relevant and sufficiently narrow indexing terms (e.g., "Evaluation Studies," "Intervention Studies," "Sexual Behavior"), without further focusing or exploding these terms, proved to be the most effective route for searching.

While the use of indexing terms is critical in locating relevant citations, indexing can be limited by numerous factors, including poorly written abstracts, misclassification, a lack of appropriate index terms, and lag time in indexing (Boynton et al. 139). Because of these limitations, a search devised with $\mathrm{MeSH}$ or indexing terms alone may fail to capture all essential information. Therefore, inverse searching was conducted within the title and abstract of the 75 citations to identify relevant keywords and phrases for each of the domains to expand to the automated search strategy. Examining the results produced by the test search also helped to determine which indexing and keyword terms were important to the final project search.

Once keywords and phrases were identified, two techniques - truncation and proximity were used to refine this aspect of the automated search. Truncation (expressed as "\$” in OVID) was used in many cases to find all spelling variants of a root term. However, using truncation alone sometimes 
retrieved an unmanageable number of citations. To narrow the search, specific keyword phrases were used when needed (i.e., "controlled trial," "control group"). Proximity operators for concept and phrase searching ("ADJ" in OVID) were used to identify two keywords near each other or variations in a particular phrase (e.g., "infect\$ adj4 rate\$"). After all aspects of the automated search were identified (electronic databases, indexing terms, keywords, and phrases), and after applying all search refinements (subheadings, EXP, focus, truncation, proximity) for each of the three key domains of the PRS project, the automated search strategy was finalized using Boolean operators to combine elements within domains ("OR") and crossreferencing between domains ("AND"). (See the complete PRS automated search strategies in Appendixes A-C.)

\section{Keeping the Search Up to Date}

An important aspect of the PRS project is keeping the internal database current. Due to the volume of citations retrieved with each search, it is only possible to update the search annually to identify the most recent literature. However, the annual search is completed for a three-year time period - one initial search for each specific year, and two subsequent searches in following years. For instance, the annual search in 2006 included publications for years 2004, 2005, and 2006. This search provided the second update for 2004 publications, the first 2005 update, and the initial 2006 search. To ensure consistency over time, the database indexing terms are regularly checked for additions, deletions, changes, or replacements. Changes in subject jargon and vernacular also evolve with time. Any needed adjustments to indexing terms or subject language are then incorporated into the search strategy, so that retrieval of the literature is kept up-to-date (Brettle et al. "Searching" 166).

\section{Manual Search}

Three manual search methods were used to complement the automated search component and to provide additional search coverage for a comprehensive search strategy. While a time-consuming endeavor, hand searching has proved to be a more direct and explicit way of retrieving relevant articles omitted by databases due to indexing inaccuracies or because of lag time in the indexing process (Avenell, Handoll, and Grant 509; Counsell 386; Jadad, Moher, and Klassen 813). A manual review of the issues from 35 key journals is conducted biannually to identify citations relevant to the focus of the PRS project. The key journals are selected by querying the PRS database for those journals producing the greatest number of HIV behavioural intervention citations with behavioural or biologic outcomes. Because this number may vary across publication year, the journal selection is re-evaluated annually. (Appendix D contains the list of journals currently searched). The second manual search method involves networking with researchers to obtain relevant published and unpublished reports. Finally, the PRS Project team continuously checks other sources to identify additional citations. These include relevant electronic mail lists, clinical trial databases (e.g., Cochrane Library, CRISP database), conference proceedings, and reference lists of relevant HIV behavioural prevention research literature.

\section{Assessing the Search}

Downloading Citations Into a General Database (PRS)

All automated and manual searches are conducted by year and by database (e.g., MEDLINE 2002). This approach narrows the number of citations to a manageable number for checking and downloading. The citations 
identified from the same database in a specific year are assigned to a unique batch number, thus allowing the examination of the contribution of each database by year. Using the OVID platform, the citations are downloaded in batches from the each electronic database to Biblioscape Reference Information Manager ${ }^{\mathrm{TM}}$ software. The PRS database is designed to check for duplications between imported citations and those already existing in the database. This process identifies the number of times a citation is found and in which database, leading to a calculation of the percentage of overlap between databases.

\section{Calculation of Search Component Contributions}

Using the batch number information, three indexes were calculated to evaluate the contributions of each search component (i.e., automated versus manual searches) and of each electronic database. These indices quantified overall contribution, unique contribution, and overlap. The indices are defined below for a search of two electronic databases, where $\boldsymbol{a}$ and $\boldsymbol{b}$ represent the number of citations found in database $A$ and $\mathrm{B}$, respectively, and where $c$ is the number of citations found in both databases (or the overlap).

The overall contribution (\%) of electronic database $\mathrm{A}$ is calculated as:

$$
\left[\frac{a}{(a+b-c)}\right] \times 100
$$

The unique contribution (\%) of electronic database A is calculated as:

$$
\left[\frac{(a-c)}{(a+b-c)}\right] \times 100
$$

The percent overlap between electronic databases A and B is calculated as:

$$
\left[\frac{c}{(a+b-c)}\right] \times 100
$$

These same formulas were used when comparing manual to automated methods and were generalized when comparing three or more electronic databases.

\section{Results}

As of December 2006, after completing the comprehensive search strategy for the years 1988-2005, a total of 17,493 citations have been identified via automated searches of the electronic databases MEDLINE, EMBASE, PsycINFO, and Sociological Abstracts (Table 1). The manual search component, including hand searching journals, reference lists, electronic mail lists, and networking with researchers, have identified 1,232 citations. After checking for overlap between automated and manual search results, there were 615 citations unique to the manual search component. The comprehensive systematic search from 1988-2005 identified a combined total of 18,108 citations. AIDSLINE identified an additional 302 citations for the years 19882000 , but these citations are not included in the calculations below. 


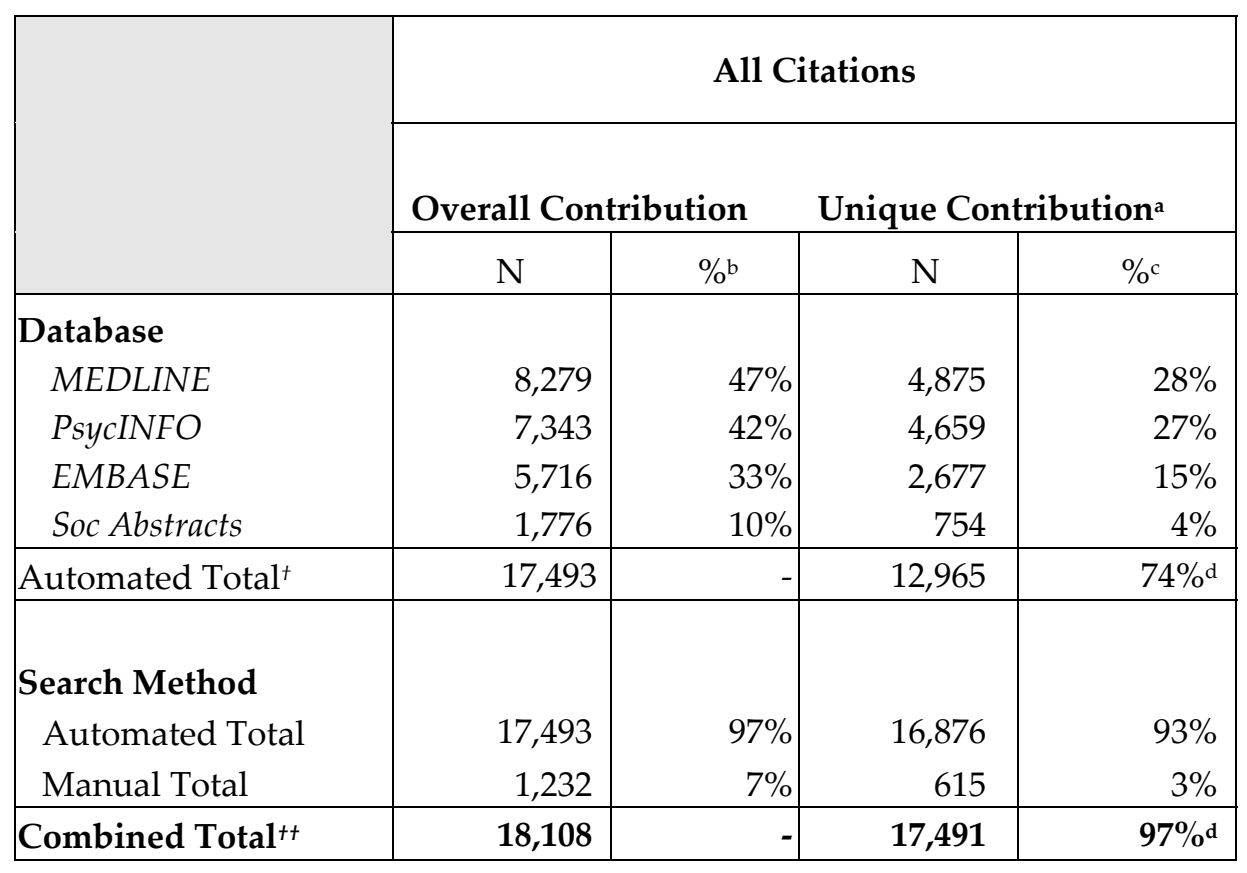

Table 1. All Citations Retrieved by Database and Method, 1988-2005

a Citations that were identified by only one database or method.

$\mathrm{b}$ Denominator is the overall contribution total. Combined percentages total more than $100 \%$ due to overlap.

c Denominator is the overall contribution total. Combined percentages total less than $100 \%$ due to overlap.

$\mathrm{d}$ Percent of total citations that were identified by only one database or method.

t The same citation was identified in 2 or more databases resulting in multiple records of the same citation. Duplicated records were removed from overall contribution totals. For all citations, 4,528 citations (26\%) were identified by 2 or more databases, resulting in 5,621 duplicate records. t+ The same citation was identified by both methods, resulting in duplicate records that are not reflected in the combined total. For all citations, 617 citations (3\%) were identified by both methods. 
Overall Contribution of each Electronic Database

Among electronic databases from the automated search component, MEDLINE, PsycINFO, and EMBASE generated the greatest retrieval of all citations $(\mathrm{n}=8,279$, $47 \% ; n=7,343,42 \%$; and $n=5,716,33 \%$, respectively, of 17,493 citations) (Table 1). Sociological Abstracts contributed $10 \%$ $(n=1,776)$ of the total number of citations identified by the automated search component. This pattern for overall contribution remained similar for particular types of citations, where MEDLINE, PsycINFO, and EMBASE continued to identify the most in-scope citations (44\%, $55 \%, 33 \%$, respectively) (Table 2 ) and behavioural/biologic citations (65\%,50\%, $46 \%$, respectively) (Table 3). PsycINFO identified the greatest number of in-scope citations ( $\mathrm{n}=5,085,55 \%) ;$ MEDLINE identified the greatest number of behavioural/biologic citations ( $n=712,65 \%)$; and Sociological Abstracts identified the fewest of both (14\% and 10\%, respectively).

\section{Unique Contribution of Each Electronic Database}

Of the 17,493 citations identified from the automated search, $74 \%(n=12,965)$ were identified by only one electronic database (Table 1 ). In other words, only $26 \%$ of the citations were identified by two or more databases. When considering the citations more specific to the PRS' primary focus (i.e., behavioural/biologic citations), a large portion (48\%) was still identified by only one database.

The unique contributions were greatest for MEDLINE and PsycINFO across all citation types: $28 \%(n=4,875)$ of all citations were identified by MEDLINE alone, and $27 \%$ $(\mathrm{n}=4,659)$ were identified only by PsycINFO (Table 1). EMBASE alone accounted for $15 \%$ $(\mathrm{n}=2,677)$ of all citations, and Sociological Abstracts alone accounted for $4 \%(n=754)$. The unique contribution for in-scope citations was greatest for PsycINFO (31\%); the unique contribution for behavioural/biologic citations was greatest for MEDLINE (20\%). Although most of the behavioural/biologic citations were identified by either MEDLINE or PsycINFO, another $10 \%$ were identified only by EMBASE (8\%), or by Sociological Abstracts (2\%).

Percent Overlap among Electronic Databases

Since MEDLINE, PsycINFO, and EMBASE contributed the greatest number of citations across all citation types, these databases were used to examine overlap. The percent overlap was calculated for each pair-wise comparison as well as for the overlap across all three electronic databases (Table 4). The percent overlap between MEDLINE and PsycINFO was $12 \%$ and varied by year. A similar percent overlap was observed between EMBASE and PsycINFO (11\% overall. Interestingly, even though MEDLINE and EMBASE both contain a biomedical focus, the percent overlap was only $20 \%$, which is only one-fifth of a total 11,697 citations identified from either database. When searching all three electronic databases, the percent overlap was $23 \%$, where $19 \%$ of the citations were identified in 2 of the 3 databases and only $4 \%$ were identified in all 3 databases (Figure 1a). 


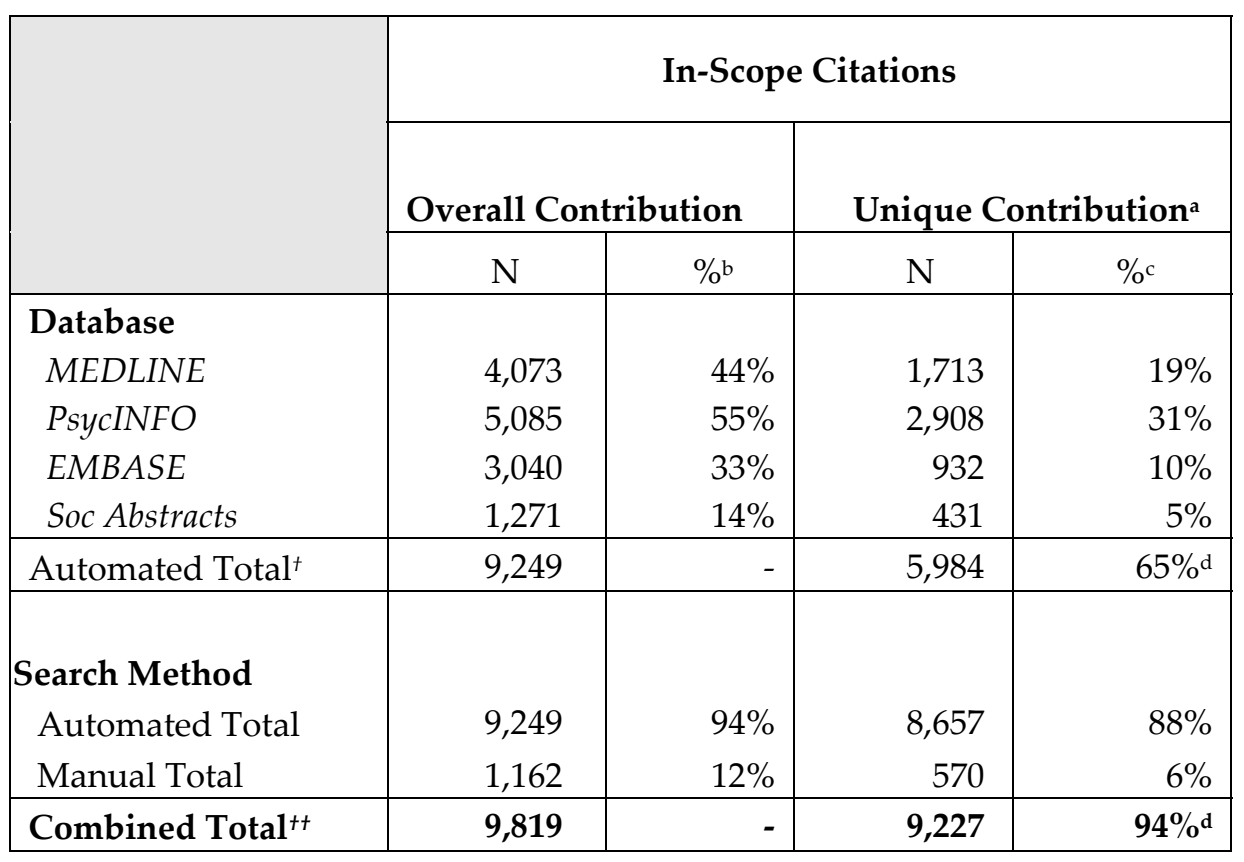

Table 2. In-Scope Citations by Database and Method, 1988-2005

a Citations that were identified by only one database or method.

$\mathrm{b}$ Denominator is the overall contribution total. Combined percentages total more than $100 \%$ due to overlap.

c Denominator is the overall contribution total. Combined percentages total less than $100 \%$ due to overlap.

$\mathrm{d}$ Percent of total citations that were identified by only one database or method.

$t$ The same citation was identified in 2 or more databases resulting in multiple records of the same citation. Duplicated records were removed from overall contribution totals. For in-scope citations, 3,265 citations (35\%) were identified by 2 or more databases, resulting in 4,220 duplicate records.

$t+$ The same citation was identified by both methods, resulting in duplicate records that are not reflected in the combined total. For in-scope citations there were $592(6 \%)$ citations identified. 


\begin{tabular}{|c|c|c|c|c|}
\hline & & $\begin{array}{l}\text { ention } \\
\text { ural/B }\end{array}$ & $\begin{array}{l}\text { Citations } \\
\text { ologic Out }\end{array}$ & \\
\hline & Overall & tion & Unique $C$ & on ${ }^{a}$ \\
\hline & $\mathrm{N}$ & $\%$ b & $\mathrm{N}$ & $\%{ }^{c}$ \\
\hline Database & & & & \\
\hline MEDLINE & 712 & $65 \%$ & 218 & $20 \%$ \\
\hline PsycINFO & 548 & $50 \%$ & 200 & $18 \%$ \\
\hline EMBASE & 508 & $46 \%$ & 91 & $8 \%$ \\
\hline Soc Abstracts & 110 & $10 \%$ & 21 & $2 \%$ \\
\hline Automated Total ${ }^{+}$ & 1,097 & - & 530 & $48 \%^{d}$ \\
\hline Search Method & & & & \\
\hline Automated Total & 1,097 & $91 \%$ & 906 & $75 \%$ \\
\hline Manual Total & 300 & $25 \%$ & 109 & $9 \%$ \\
\hline Combined Total ${ }^{++}$ & 1,206 & - & 1,015 & $84 \%{ }^{d}$ \\
\hline
\end{tabular}

Table 3. Intervention Citations by Database and Method, 1988-2005

a Citations that were identified by only one database or method.

$\mathrm{b}$ Denominator is the overall contribution total. Combined percentages total more than $100 \%$ due to overlap.

c Denominator is the overall contribution total. Combined percentages total less than $100 \%$ due to overlap.

d Percent of total citations that were identified by only one database or method t The same citation was identified in 2 or more databases, resulting in multiple records of the same citation. Duplicated records were removed from overall contribution totals. For intervention citations with behavioural or biologic outcomes, 567 citations (52\%) were identified by 2 or more databases, resulting in 781 duplicate records.

t+ The same citation was identified by both methods, resulting in duplicate records not reflected in the combined total. For intervention citations with behavioural or biologic outcomes, 191 (16\%) citations were identified. 


\begin{tabular}{|c|c|c|c|c|c|c|c|c|}
\hline & \multicolumn{4}{|c|}{ All Citations ${ }^{a}$} & \multicolumn{4}{|c|}{$\begin{array}{c}\text { Intervention Citations with } \\
\text { Behavioural/Biologic Outcomes }^{\text {b }}\end{array}$} \\
\hline & \multirow[b]{2}{*}{ Total } & \multirow{2}{*}{$\begin{array}{l}\text { Uniquely } \\
\text { Identified }^{c}\end{array}$} & \multicolumn{2}{|c|}{ Overlap } & \multirow[b]{2}{*}{ Total } & \multirow{2}{*}{$\begin{array}{c}\text { Uniquely } \\
\text { Identified }^{c}\end{array}$} & \multicolumn{2}{|c|}{ Overlap } \\
\hline & & & $\mathbf{N}$ & $\%$ & & & $\mathbf{N}$ & $\%$ \\
\hline MEDLINE \& PsycINFO & 13,937 & 12,252 & 1,685 & $12 \%$ & 977 & 694 & 283 & $29 \%$ \\
\hline MEDLINE \& EMBASE & 11,697 & 9,399 & 2,298 & $20 \%$ & 861 & 502 & 359 & $42 \%$ \\
\hline EMBASE \& PsycINFO & 11,764 & 10,469 & 1,295 & $11 \%$ & 847 & 638 & 209 & $25 \%$ \\
\hline $\begin{array}{l}\text { MEDLINE, PsycINFO, } \\
\& \text { EMBASE }\end{array}$ & 16,739 & 12,819 & $3,920^{*}$ & $23 \%$ & 1,076 & 543 & $533^{* *}$ & $50 \%$ \\
\hline
\end{tabular}

Table 4. Overlap of Citations between MEDLINE, PsycINFO, and EMBASE, Searching the 19882005 Literature

a The number of citations identified by each database: $\operatorname{MEDLINE}(\mathrm{n}=8,279)$,

PsycINFO (n=7,343), EMBASE (n=5,716)

$\mathrm{b}$ The number of intervention citations with behavioural/biologic outcomes identified by each database: MEDLINE ( $\mathrm{n}=712), P s y c I N F O(\mathrm{n}=548)$, EMBASE $(\mathrm{n}=508)$

c Citations identified by either one, but only one, database

* 3,241 citations (19\%) were identified in 2 of the 3 databases; $679(4 \%)$ were identified in all 3

** 374 citations (35\%) were identified in 2 of the 3 databases; $159(15 \%)$ were identified in all 3 
For behavioural/biologic citations, the percent overlap between databases increased substantially compared to the percent observed among all citations (Table 4). The greatest overlap was again between MEDLINE and EMBASE (42\%). The percent overlap between MEDLINE and PsycINFO was $29 \%$, and between EMBASE and $P$ sycINFO the overlap was $25 \%$. The percent overlap for behavioural/biologic citations among all 3 databases was 50\%, where $35 \%$ of the citations were identified in 2 of the 3 databases. Only $15 \%$ were identified in all 3 databases (Figure 1b).

Figure 1. Overlap of MEDLINE, EMBASE, and PsycINFO, Searching the 1988-2005 Literature

Figure 1a. All Citations

$(\mathrm{N}=16,739)$

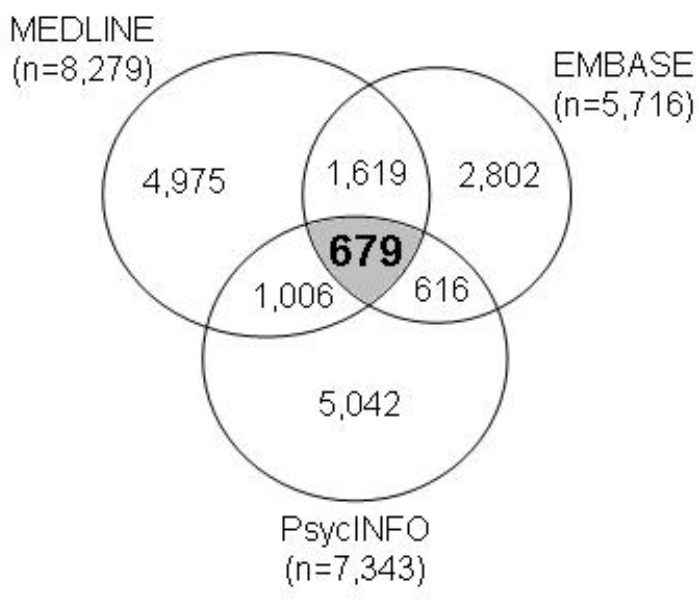

Figure 1b. Intervention Citations with Behavioural or Biologic Outcomes $(\mathrm{N}=1,076)$

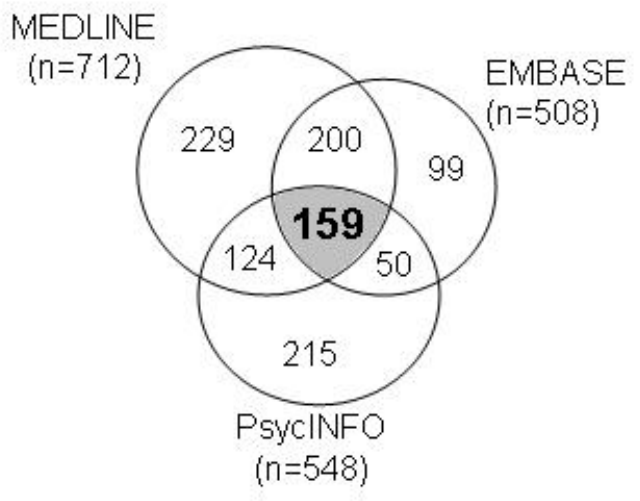


Contribution of the Search Methods: Manual and Automated

Overall, the manual search method added $7 \%$ of the citations to the PRS database, in contrast to a contribution of $97 \%$ for the automated search method (Table 1). The contribution of the manual search method increased to $12 \%$ for in-scope citations and to $25 \%$ for behavioural/biologic citations, while the contribution for the automated search method decreased slightly to $91 \%$ for behavioural/biologic citations. The increase in overall contribution by the manual search method was accompanied by an increase in overlap with the automated search component, an increase in unique contribution of the manual search, and a decrease in unique contribution of the automated search. Of the 18,108 citations identified overall, only $3 \%$ were identified by both methods (Table 1 ). There was greater overlap for in-scope citations $(6 \%)$ (Table 2) and for behavioural/biologic citations (16\%) (Table 3). As far as unique contributions, $6 \%(n=570)$ of the in-scope citations (Table 2$)$ and $9 \%(n=109)$ of the behavioural/biologic citations (Table 3) were identified exclusively by the manual search component. These 109 uniquely identified behavioural/biologic citations, which are the primary focus of the PRS Project, would have been missed if only automated searches were conducted.

\section{Discussion}

Developing, testing, and implementing a systematic search for research synthesis is an extremely complex and time-intensive task. Librarians should be considered an integral part of the systematic review process in the evidence based healthcare field (Beverley, Booth, and Bath 66; McGowan and Sampson; Sampson and McGowan 1057; Helmer et al. 347; Harris 86;
Zhang, Sampson, and McGowan 5). Although the specifics of this search strategy focus on the PRS project needs, the general framework for developing a systematic search strategy as described within this paper may be valuable to other research synthesis projects in a variety of disciplines, and it adds to the methods for search strategy development already presented (Matthews et al; McNally and Alborz). A thorough evaluation of any systematic review cannot happen without explicit documentation of the process used by researchers. Additionally, it is difficult to place confidence in the findings of a systematic review, if the systematic search used to generate the pool of literature for that review is not clearly reported (Booth 426; Sampson and McGowan 1160; Weller 163; Patrick et al. 199).

During the development, testing, and refinement of the PRS search strategy, various methods and techniques were selected to ensure the breadth and depth of the search. Using both indexing terms and keyword searching can improve the retrieval of relevant citations. These techniques complement each other and will help overcome potential differences in use of terminology among authors, as well as the limitations of indexing. The PRS project focuses on completeness of purpose, rather than precision or specificity. The effectiveness of an automated search strategy can be improved by testing the strategy and evaluating the results.

Monitoring indexing terms over time is also necessary, since databases add, change and delete terms routinely. New terms can narrow or broaden the meaning and relevance of existing terms, altering the outcome of an automated search. One potential problem is that these changes may not be applied consistently to all citations 
published prior to the changes. For example, the term "Unsafe Sex" was added as a MeSH term to MEDLINE in 2005. This term, however, was added to the indexing of citations published prior to the change, because they were not indexed until 2005. Similarly, keyword terms and concepts may also change over time, necessitating additional modification and retesting of the automated search. While the search must evolve as new terms enter the subject matter vernacular, it is also important to be aware of the potential issues associated with any modification.

Consistent with previous findings (Alpi 99; Avenell, Handoll, and Grant 508; Brettle and Long 354; Minozzi, Pistotti, and Forni 721), examinations of the overlap between databases and the unique contributions of each database suggest that multiple databases should be used for systematic searching. In addition, the automated search should be supplemented by a manual search component, including a hand search of journals, conference proceedings and abstracts, reference chasing, and personal communications with key researchers in the field. A combination of these two approaches will increase the likelihood of capturing all the relevant citations for research synthesis activities.

It is important to point out that the amount of overlap between databases and the unique contributions of each database presented here should be interpreted with caution. This evaluation was performed within the context of the HIV behavioural prevention research literature and may not be generalizable to other research fields. It is likely that systematic searches in other behavioural science or social science fields could produce similar findings and conclusions; however, further testing would be required to make that determination. It is also inappropriate to use this information to select a database with the highest yield for sole use in conducting a systematic review. Within the HIV behavioural prevention research field, all four electronic databases (MEDLINE, EMBASE, PSycINFO, and Sociological Abstracts) and the manual search component made important unique contributions to the retrieval of relevant literature.

\section{Conclusion}

As the HIV prevention field becomes increasingly more reliant on systematic reviews of the literature for making evidence based recommendations, it becomes ever more critical to improve the quality of searching the research literature. Developing and implementing a systematic and comprehensive search strategy for identifying all the relevant research literature is a vital first step in conducting a systematic review. Creating a search strategy requires a well thought out process for planning, testing, and refining, but this process is necessary to ensure that all relevant information is included in any research synthesis activity. These standards are important in moving evidence based public health and information practice forward.

\section{Acknowledgments}

The findings and conclusions in this report are those of the authors and do not necessarily represent the views of the Centers for Disease Control and Prevention. This work was supported by the Prevention Research Branch, Division of HIV/AIDS Prevention, US Centers for Disease Control and Prevention and was not funded by any other organisation. The authors thank past and present members of the CDC's HIV/AIDS Prevention Research Team Tanesha Griffin, Jeffrey H. Herbst, Angela K. Horn, Angela Hutchinson, Elizabeth D. Jacobs, Laura V. Lloyd, Paola MarreroGonzalez, Warren Passin, Jocelyn Patterson, 
Sima Rama, R. Thomas Sherba, Lev

Zohrabyan - who contributed to the development and maintenance of the PRS database.

\section{Works Cited}

Alpi, Kristine M. "Expert Searching in Public Health." Journal of the Medical Library Association 93.1 (Jan. 2005): 97-103.

Atkins, David, Kenneth Fink, Jean Slutsky, and the Agency for Healthcare Research and Quality; North American Evidence-based Practice Centers. "Better Information for Better Health Care: The EvidenceBased Practice Center Program and the Agency for Healthcare Research and Quality." Annals of Internal Medicine 142.12 Pt. 2 (21 June 2005): 1035-41.

Avenell, Alison, Helen G. Handoll, and Adrian M. Grant. "Lessons for Search Strategies from a Systematic Review, in the Cochrane Library, of Nutritional Supplementation Trials in Patients After Hip Fracture." American Journal of Clinical Nutrition 73.3 (Mar. 2001): 505-10.

Berry, Elizabeth, Steven Kelly, John Hutton, Keith M. Harris, and Michael A. Smith. "Identifying Studies for Systematic Reviews. An Example from Medical Imaging." International Journal of Technology Assessment in Health Care 16.2 (Spring 2000): 668-72.

Beverley, Catherine, Andrew Booth, and Peter A. Bath. "The Role of the Information Specialist in the Systematic Review Process: A
Health Information Case Study." Health Information and Libraries Journal 20.2 (June 2003): 65-74.

Biblioscape Reference Information Manager $^{\mathrm{TM}}$. Vers. 6.6. June 2007 $<$ http://www.biblioscape.com/index. html>.

Booth, Andrew. "Brimful of STARLITE": Toward Standards for Reporting Literature Searches." Journal of the Medical Library Association 94.4 (Oct. 2006): 421-9, e205.

Boynton, Janette, Julie Glanville, David McDaid, and Carol Lefebvre. "Identifying Systematic Reviews in MEDLINE: Developing an Objective Approach to Search Strategy Design." Journal of Information Science 24.3 (1 June 1998): 137-57.

Brettle, Alison J., and Andrew F. Long. "Comparison of Bibliographic Databases for Information on the Rehabilitation of People with Severe Mental Illness." Bulletin of the Medical Library Association 89.4 (Oct. 2001): 353-62.

Brettle, Alison J., Andrew F. Long, Maria J. Grant, and Joanne Greenhalgh. "Searching for Information on Outcomes: Do You Need to be Comprehensive?" Quality in Health Care 7.3 (Sept. 1998): 163-7.

Bruce, Julie, and Jill Mollison. "Reviewing the Literature: Adopting a Systematic Approach." Journal of Family Planning and Reproductive Health Care 30.1 (2004): 13-6.

Chalmers, Ian, Kay Dickersin, and Thomas C. Chalmers. "Getting to Grips with Archie Cochrane's Agenda." BMI 305.6857 (3 Oct. 1992): 786-8. 
Collins, Charles, Camilla Harshbarger, Richard Sawyer, and Myriam Hamdallah. "The Diffusion of Effective Behavioral Interventions Project: Development, Implementation, and Lessons Learned." $\underline{\text { AIDS Education and }}$ Prevention 18.4 Suppl. A (Aug. 2006): 5-20.

Conn, Vicki S., Sang-arun Isaramalai, Sabyasachi Rath, Peeranuch Jantarakupt, Rohini Wadhawan, and Yashodhara Dash. "Beyond MEDLINE for Literature Searches." Journal of Nursing Scholarship 35.2 (2003): 177-82.

Counsell, Carl. "Formulating Questions and Locating Primary Studies for Inclusion in Systematic Reviews." Annals of Internal Medicine 127.5 (Sept. 1997): 380-7.

Crepaz, Nicole, Warren F. Passin, Jeffrey H. Herbst, Sima M. Rama, Robert M. Malow, David W. Purcell, and Richard J. Wolitski. "Meta-Analysis of Cognitive-Behavioral Interventions on HIV-Positive Persons' Mental Health and Immune Functioning." Health Psychology 27.1 (2008): 4-14.

Crepaz, Nicole, Cynthia M. Lyles, Richard J, Wolitski, Warren F. Passin, Sima M. Rama, Jeffrey H. Herbst, David W. Purcell, Robert M. Malow, Ron Stall, and the HIV/AIDS Prevention Research Synthesis Team. "Do Prevention Interventions Reduce HIV Risk Behaviours Among People Living with HIV? A Meta-Analytic Review of Controlled Trials." AIDS 20.2 (9 Jan. 2006): 143-57.

Crepaz, Nicole, Angela K. Horn, Sima M. Rama, Tanesha Griffin, Julia B.
DeLuca, Mary M. Mullins, Sevgi O. Aral, and the HIV/AIDS Prevention Research Synthesis Team. "The Efficacy of Behavioral Interventions in Reducing HIV Risk Sex Behaviors and Incident Sexually Transmitted Disease in Black and Hispanic Sexually Transmitted Disease Clinic Patients in the United States: A Meta-Analytic Review." Sexually Transmitted Diseases 34.6 (June 2007): 319-32.

Crumley, Ellen T., and Karen Blackhall. "Setting Up Search Strategies for Systematic Reviews (Or, How Many Ways Can You Spell Diarrhea?)." Bibliotheca Medica Canadiana 24.4 (2003): 167-8.

Crumley, Ellen T., Natasha Wiebe, Kritie Cramer, Terry P. Klassen, and Lisa Hartling. "Which Resources Should be Used to Identify RCT/CCTs for Systematic Reviews: A Systematic Review." BMC Medical Research Methodology 5.24 (10 Aug. 2005). doi: 10.1186/1471-2288-5-24

Dickersin, Kay, Renslow Scherer, and Carol Lefebvre. "Identifying Relevant Studies for Systematic Reviews." BMJ 309.6964 (12 Nov. 1994): 128691.

"Evidence-Based Interventions." Centers for Disease Control and Prevention. 2007. HIV/AIDS Prevention Research Synthesis Project (PRS). 13 Oct. 2007.

<http://www.cdc.gov/hiv/topics/res earch/prs/evidence-basedinterventions.htm>.

"FAQ: Journal Selection for MEDLINE® Indexing at NLM." National Library of Medicine. 2007 National Library of Medicine, Bethesda, MD. 13 Oct. 
2007.

<http://www.nlm.nih.gov/pubs/fact sheets/j sel faq.html $>$.

Greenhalgh, Trisha. "How to Read a Paper. The Medline Database." BMI 315.7101 (19 July 1997): 180-3.

Harris, Martha R. "The Librarian's Roles in the Systematic Review Process: A Case Study." Journal of the Medical Library Association 93.1 (Jan. 2005): 81-7.

Helmer, Diane, Isabellel Savoie, Carolyn Green, and Arminée Kazanjian. "Evidence-Based Practice: Extending the Search to Find Material for the Systematic Review." Bulletin of the Medical Library Association 89.4 (Oct. 2001): 346-52.

Herbst, Jeffrey H., R. Thomas Sherba, Nicole Crepaz, Julia B. DeLuca, Lev Zohrabyan, Ron D. Stall, Cynthia M. Lyles, and the HIV/AIDS Prevention Research Synthesis Team. "A MetaAnalytic Review of HIV Behavioral Interventions for Reducing Sexual Risk Behavior of Men Who Have Sex with Men." IAIDS Journal of Acquired Immune Deficiency Syndromes 39.2 (1 June 2005): 22841.

Herbst, Jeffrey H., Linda S. Kay, Warren F. Passin, Cynthia M. Lyles, Nicole Crepaz, Barbara V. Marín, and the HIV/AIDS Prevention Research Synthesis Team. "A Systematic Review and Meta-Analysis of Behavioral Interventions to Reduce HIV Risk Behaviors of Hispanics in the United States and Puerto Rico." AIDS and Behavior 11.1 (Jan. 2007): 25-47.
Herbst, Jeffrey H., Carolyn Beeker, Anita Mathew, Tarra McNally, Warren F. Passin, Linda S. Kay, Nicole Crepaz, Cynthia M. Lyles, Peter Briss, Sajal Chattopadhyay, Robert L. Johnson, and the Task Force on Community Preventive Services. "The Effectiveness of Individual-, Group-, and Community-Level HIV Behavioral Risk-Reduction Interventions for Adult Men Who Have Sex With Men: A Systematic Review." American Journal of Preventive Medicine 32.4 Suppl. 1 (Apr. 2007): S38-S67.

Higgins, Julian P., and Sally Green eds. Cochrane Handbook for Systematic Reviews of Interventions 4.2.6 [Updated September 2006].. 24 February 2008 $<$ http://www.cochrane.org/resource s/handbook/index.htm>.

Howes, Faline, Jodie Doyle, Nicki Jackson, and Elizabeth Waters. "EvidenceBased Public Health: The Importance of Finding 'Difficult to Locate' Public Health and Health Promotion Intervention Studies for Systematic Reviews." Journal of Public Health 26.1 (Mar. 2004): 1014.

Jadad, Alejandro R., David Moher, and Terry P. Klassen. "Guides for Reading and Interpreting Systematic Reviews: II. How Did the Authors Find the Studies and Assess Their Quality?" Archives of Pediatrics and Adolescent Medicine 152.8 (Aug. 1998): 812-7.

Jenuwine, Elizabeth S., and Judith A. Floyd. "Comparison of Medical Subject Headings and Text-Word Searches in MEDLINE to Retrieve Studies on Sleep in Healthy Individuals." 
Journal of the Medical Library

Association 92.3 (July 2004): 349-53.

Johnson, Blair T., Michael P. Carey, Stephanie Chaudoir, and Allecia E. Reid. "Sexual Risk Reduction for Persons Living with HIV: Research Synthesis of Randomized Controlled Trials, 1993 to 2004." Journal of Acquired Immune Deficiency Syndromes 41.5 (15 Apr. 2006): 642-50.

Lyles, Cynthia M., Linda S. Kay, Nicole Crepaz, Jeffrey H. Herbst, Warren F. Passin, Angela S. Kim, Sima M. Rama, Sekhar Thadiparthi, Julia B. DeLuca, Mary M. Mullins, and the HIV/AIDS Prevention Research Synthesis Project Team. "BestEvidence Interventions: Findings from a Systematic Review of HIV Behavioral Interventions for U.S. Populations at High Risk, 20002004." American Journal of Public Health 97.1 (Jan. 2007): 133-43.

Lyles, Cynthia M., Nicole Crepaz, Jeffrey H. Herbst, Linda S. Kay, and the HIV/AIDS Prevention Research Synthesis Project Team. "EvidenceBased HIV Behavioral Prevention from the Perspective of the CDC's HIV/AIDS Prevention Research Synthesis Team." AIDS Education and Prevention 18.4 Suppl. A (Aug. 2006): 21-31.

Matthews, Elaine J., Adrian G. K. Edwards, Jackie Barker, Michael Bloor, Judith Covey, Kerenza Hood, Roisin Pill, IaN. Russell, Nigel Stott, and Clare Wilkinson. "Efficient Literature Searching in Diffuse Topics: Lessons from a Systematic Review of Research on Communicating Risk to Patients in Primary Care." Health Libraries Review 16.2 (1999): 112-20.
20 Feb. 2008. doi:10.1046/j.13652532.1999.00219.x

McGowan, Jessie, and Margaret Sampson. "Systematic Reviews Need Systematic Searchers." Journal of the Medical Library Association 93.1 (Jan. 2005): 74-80.

McManus, Richard J., Sue Wilson, Brendan C. Delaney, David A. Fitzmaurice, C. J. Hyde, R. S. Tobias, S. Jowett, and Richard Hobbs. "Review of the Usefulness of Contacting Other Experts When Conducting a Literature Search for Systematic Reviews." BMI 317.7172 (5 Dec. 1998): 1562-3.

McNally, Rosalind, and Alison Alborz.

"Developing methods for systematic reviewing in health services delivery and organization: an example from a review of access to health care for people with learning disabilities. Part 1. Identifying the literature." Health Information and Libraries Journal 21.3 (Sept. 2004): 182-92.

Minozzi, Silvia, Vanna Pistotti, and Marco Forni. "Searching for Rehabilitation Articles on MEDLINE and EMBASE. An Example with Cross-Over Design." Archives of Physical Medicine and Rehabilitation 81.6 (June 2000): 720-2.

Neumann, Mary Spink, and Ellen Sogolow. "Replicating Effective Programs: HIV/AIDS Prevention Technology Transfer." AIDS Education and Prevention 12.Suppl. 5 (2000): 35-48.

Passin, Warren F., Angela S. Kim, Angela B. Hutchinson, Nicole Crepaz, Jeffrey H. Herbst, Cynthia M. Lyles, and the HIV/AIDS Prevention Research 
Synthesis Project Team. "A

Systematic Review of HIV Partner

Counseling and Referral Services:

Client and Provider Attitudes,

Preferences, Practices, and

Experiences." Sexually Transmitted

Diseases 33.5 (May 2006): 320-8.

Patrick, Timothy B., George Demiris, Lillian

C. Folk, David E. Moxley, Joyce A.

Mitchell, and Donghua Tao.

"Evidence-Based Retrieval in

Evidence-Based Medicine." Journal

of the Medical Library Association

92.2 (Apr. 2004): 196-9.

Perry, Gerald J., and Michael R. Kronenfeld.

"Evidence-Based Practice: A New

paradigm Brings New

Opportunities for Health Sciences

Librarians." Medical Reference

Services Quarterly 24.4 (Winter

2005): 1-16.

Peterson, Amy S., and Lisa M. Randall.

"Utilizing Multilevel Partnerships to

Build the Capacity of Community-

Based Organizations to Implement

Effective HIV Prevention

Interventions in Michigan." $\underline{\text { AIDS }}$

Education and Prevention 18.4

Suppl A (2006): 83-95.

Robinson, Karen A., and Kay Dickersin.

"Development of a Highly Sensitive Search Strategy for the Retrieval of Reports of Controlled Trials Using PubMed." International Journal of Epidemiology 31.1 (2002): 150-3.

Sackett, David L., William M. C. Rosenberg, J. A. Muir Gray, R. Brian Haynes, and W. Scott Richardson. "Evidence Based Medicine: What It Is and What It Isn't." BMI 312.7023 (13 Jan. 1996): 71-2.
Sampson, Margaret and Jessie McGowan. "Errors in Search Strategies Were Identified By Type and Frequency." Journal of Clinical Epidemiology 59.10 (2006): 1057-63.

Savoie, Isabelle, Diane Helmer, Carolyn J. Green, and Arminee Kazanjian. "Beyond Medline: Reducing Bias Through Extended Systematic Review Search." International Journal of Technology Assessment in Health Care 19.1 (Winter 2003): 168-78.

Shea, Madeline A., Barry P. Callis, Hope Cassidy-Stewart, Kevin Cranston, and Naomi Tomoyasu. "Diffusion of Effective HIV Prevention Interventions--Lessons From Maryland and Massachusetts." AIDS Education and Prevention 18.4 Suppl A (Aug. 2006): 96-107.

Simoni, Jane M., Cynthia R. Pearson, David W. Pantalone, Gary Marks, and Nicole Crepaz. "Efficacy of Interventions in Improving Highly Active Antiretroviral Therapy Adherence and HIV-1 RNA Viral Load: A Meta-Analytic Review of Randomized Controlled Trials." Journal of Acquired Immune Deficiency Syndromes 43.Suppl 1 (Dec. 2006): S23-S35.

Sogolow, Ellen, Greet Peersman, Salaam Semaan, Darcy Strouse, Cynthia M. Lyles, and the HIV/AIDS Prevention Research Synthesis Project Team. "The HIV/AIDS Prevention Research Synthesis Project: Scope, Methods, and Study Classification Results." Journal of Acquired Immune Deficiency Syndromes 30.Suppl. 1 (2002): S15-S29. 
Victora, Cesar G., Jean-Pierre Habicht, and Jennifer Bryce. "Evidence-Based Public Health: Moving Beyond Randomized Trials." American Journal of Public Health 94.3 (2004): 400-5.

Watson, R. J., and Philip H. Richardson. "Identifying Randomized Controlled Trials of Cognitive Therapy for Depression: Comparing the Efficiency of EMBASE, Medline and PsycINFO Bibliographic Databases." British Journal of Medical Psychology 72.Pt. 4 (1999): $535-42$.

Weller, Ann C. "Mounting Evidence That Librarians Are Essential for Comprehensive Literature Searches for Meta-Analyses and Cochrane Reports." Journal of the Medical Library Association 92.2 (Apr. 2004): 163-4.

Zhang, Li, Margaret Sampson, and Jessie MacGowan. "Reporting of the Role of the Expert Searcher in Cochrane Reviews." Evidence Based Library and Information Practice 1.4 (Dec. 2006): 3-16. 18 Feb. 2008 $<$ http://ejournals.library.ualberta.ca/ index.php/EBLIP/article/view/57/15 7>. 


\section{Appendix A - OVID Search Strategy - MEDLINE \\ $\$=$ truncation $\quad *$ focus \\ $\mathrm{ab}=$ abstract $\quad \mathrm{ti}=$ title \\ /ut $=$ utilization subheading \\ $/ \mathrm{pc}=$ prevention and control subheading}

\section{HIV/AIDS/STDs MeSH}

\begin{tabular}{|c|c|}
\hline 1. & ${ }^{*}$ HIV Infections/pc \\
\hline 2 & ${ }^{*} \mathrm{AIDS} / \mathrm{pc}$ \\
\hline & *Sexually Transmitted \\
\hline & Diseases/pc \\
\hline T. & $\begin{array}{l}\text { *Sexually Transmitted Diseases, } \\
\text { bacterial/ }\end{array}$ \\
\hline & $\begin{array}{l}\text { *Sexually Transmitted diseases, } \\
\text { viral/ }\end{array}$ \\
\hline & AIDS serodiagnosis/ut \\
\hline & ${ }^{*}$ HIV Seropositivity/ \\
\hline & or/1-7 \\
\hline
\end{tabular}

\section{Prevention/Intervention MeSH and Keywords}

$\begin{array}{ll}\text { 9. } & \text { Primary Prevention/ } \\ \text { 10. } & \text { Preventive Health Services/ } \\ \text { 11. } & \text { Health Promotion/ } \\ \text { 12. } & \text { Program Evaluation/ } \\ \text { 13. } & \text { Randomized Controlled Trials/ } \\ \text { 14. } & \text { Evaluation Studies/ } \\ \text { 15. } & \text { Contact Tracing/ } \\ \text { 16. } & \text { Case Management/ } \\ \text { 17. } & \text { Needle-Exchange Programs/ } \\ \text { 18. } & \text { Intervention Studies/ } \\ \text { 19. } & \text { Follow-Up Studies/ } \\ \text { 20. } & \text { Longitudinal Studies/ } \\ \text { 21. } & \text { Multicenter Studies/ } \\ \text { 22. } & \text { Random Allocation/ } \\ \text { 23. } & \text { (control group).ti,ab } \\ \text { 24. } & \text { (control trial).ti,ab } \\ \text { 25. } & \text { (controlled trial).ti,ab } \\ \text { 26. } & \text { (rct or rcts).ti,ab } \\ \text { 27. } & \text { (case management).ti,ab }\end{array}$

28. (contact tracing).ti, ab

29. (counseling or counselling).ti,ab

30. (detox or detoxification).ti,ab

31. (drug\$ adj4 treatment\$).ti,ab

32. education $\$ . t i, a b$

33. (effect or effects or effective or effectiveness).ti, ab

34. efficacy.ti,ab

35. evaluation\$.ti, ab

36. impact $\$ . t i, a b$

37. intervention\$.ti, ab

38. (needle exchang\$).ti,ab

39. network\$.ti,ab

40. outreach\$.ti,ab

41. (partner notification).ti,ab

42. (partner\$ adj4 contact\$ adj4 referral\$).ti, ab

43. (notif\$ adj4 partner\$).ti,ab

44. prevention $\$ . t i, a b$

45. program $\$ . t i, a b$

46. random $\$ . t i, a b$

47. rehab\$.ti,ab

48. skill\$.ti,ab

49. (syringe exchang\$).ti,ab

50. methadone.ti, ab

51. test\$.ti,ab

52. training.ti,ab

53. trial\$.ti,ab

54. or/9-53

\section{Behavior/Outcomes MeSH and Keywords}

55. Behavior/

56. Behavior Therapy/

57. Health Behavior/

58. Risk Reduction Behavior/

59. Risk-Taking/

60. Contraception Behavior/

61. Coitus/

62. Sexual Abstinence/

63. Sexual Behavior/

64. Sexual Partners/

65. Safe Sex/

66. Unsafe Sex/

67. Heroin Dependence/pc

68. Needle Sharing/

69. Condoms/ut 
70. Condoms, female/ut

71. Contraceptive devices, male/ut

72. Contraceptive devices, female/ut

73. Substance abuse, intravenous/pc

74. Substance-related disorders/pc

75. Cocaine-related disorders/pc

76. Health services/ut

77. heroin.ti,ab

78. cocaine.ti, ab

79. opiate\$.ti,ab

80. opium.ti,ab

81. paraphernalia.ti,ab

82. (treatment\$ adj2 entry).ti,ab

83. (treatment\$ adj2 enter\$).ti,ab

84. (abstin\$ or abstain\$).ti,ab

85. drug\$.ti,ab

86. substance.ti,ab

87. (idu or idus or ivdu or ivdus).ti,ab

88. (behavior\$ or behaviour\$ or activit $\$$ or access $\$$ or utiliz $\$$ or use $\$$ or using $\$$ or test $\$$ or risk $\$$ or outcome\$) adj4 (reduc $\$$ or declin $\$$ or chang $\$$ or effect $\$$ or increas $\$$ or decreas $\$$ or impact $\$$ or modif $\$$ or lower $\$$ or maintain\$ or maintenance)).ti,ab

89. bleach $\$ . t i, a b$

90. clean\$.ti,ab

91. condom\$.ti, ab

92. contracept\$.ti,ab

93. crack.ti,ab

94. disclos\$.ti,ab

95. incidence.ti, ab

96. inject $\$ . t i, a b$

97. intention\$.ti,ab

98. intercourse.ti,ab

99. needle\$.ti, ab

100. infect $\$$ adj4 (new $\$$ or rate $\$$ or declin $\$$ or reduc $\$$ or prevent $\$$ or lower\$ or decreas\$).ti,ab

101. partner $\$ . t i, a b$

102. seroconver\$.ti, ab

103. sex\$.ti,ab

104. syring $\$ . t i, a b$

105. test\$.ti,ab
106. or/55-105

107. 8 and 54 and 106

Limits: English language, publication types, Date limits 


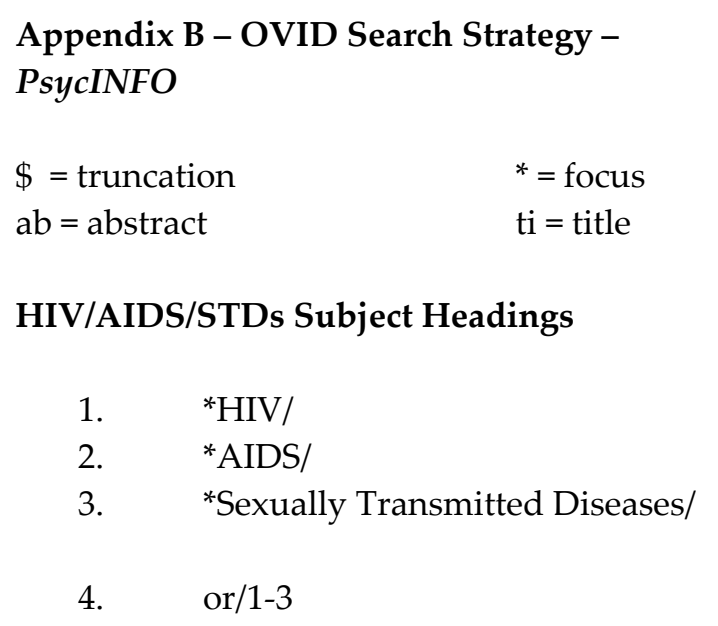

Prevention/Intervention Subject Headings and Keywords

\begin{tabular}{|c|c|}
\hline 5. & Prevention/ \\
\hline 6. & AIDS Prevention/ \\
\hline 7. & HIV Testing/ \\
\hline 8. & Primary Health Care/ \\
\hline 9. & Health Promotion/ \\
\hline 10. & Program Evaluation/ \\
\hline 11. & Case Management/ \\
\hline 12. & Needle-Exchange Programs/ \\
\hline 13. & Followup Studies/ \\
\hline 14. & Longitudinal Studies/ \\
\hline 15. & Random Sampling/ \\
\hline 16. & control group.ti,ab \\
\hline 17. & control trial.ti,ab \\
\hline 18. & controlled trial.ti,ab \\
\hline 19. & (rct or rcts).ti,ab \\
\hline 20. & case management.ti,ab \\
\hline 21. & contact tracing.ti,ab \\
\hline 22. & (counseling or counselling).ti,ab \\
\hline 23. & (detox or detoxification).ti,ab \\
\hline 24. & (drug\$ adj4 treatment\$).ti,ab \\
\hline 25. & education\$.ti,ab \\
\hline 26. & $\begin{array}{l}\text { (effect or effects or effective or } \\
\text { effectiveness).ti,ab }\end{array}$ \\
\hline 27. & efficacy.ti,ab \\
\hline 28. & evaluation $\$ . t i, a b$ \\
\hline 29. & impact\$.ti,ab \\
\hline 30. & intervention\$.ti,ab \\
\hline 31. & needle exchang\$.ti,ab \\
\hline 32. & network\$.ti,ab \\
\hline 33. & outreach\$.ti,ab \\
\hline 34. & partner notification.ti,ab \\
\hline
\end{tabular}

35. (partner $\$$ adj4 contact $\$$ adj4 referral\$).ti,ab

36. (notif\$ adj4 partner\$).ti,ab

37. prevention\$.ti,ab

38. program\$.ti,ab

39. random $\$ . t i, a b$

40. rehab\$.ti,ab

41. skill\$.ti,ab

42. syringe exchang\$.ti,ab

43. methadone.ti,ab

44. test\$.ti,ab

45. training.ti,ab

46. trial\$.ti,ab

47. or $/ 5-46$

\section{Behavior/Outcomes Subject Headings and Keywords}

\begin{tabular}{ll} 
48. & Behavior/ \\
49. & Behavior Therapy/ \\
50. & Health Behavior/ \\
51. & Risk-Taking/ \\
52. & Sexual Risk Taking / \\
53. & Sexual Abstinence/ \\
54. & Sexual Partners/ \\
55. & Safe Sex/ \\
56. & Psychosexual Behavior/ \\
57. & Behavior Change/ \\
58. & Condoms/ \\
59. & Contraceptive Devices/ \\
60. & Drug Abuse/ \\
61. & Intravenous Drug Usage/ \\
62. & Cocaine/ \\
63. & Heroin Addiction/ \\
64. & Heroin/ \\
65. & Needle Sharing/ \\
66. & At Risk Populations/ \\
67. & heroin.ti,ab \\
68. & cocaine.ti,ab \\
69. & opiate\$.ti,ab \\
70. & opium.ti,ab \\
71. & paraphernalia.ti,ab \\
72. & (treatment\$ adj2 entry).ti,ab \\
73. & (treatment\$ adj2 enter\$).ti,ab \\
74. & (abstin $\$$ or abstain\$).ti,ab \\
75. & drug\$.ti,ab \\
76. & substance.ti,ab \\
\hline &
\end{tabular}


77. (idu or idus or ivdu or ivdus).ti,ab

78. ((behavior\$ or behaviour\$ or activit $\$$ or access $\$$ or utiliz $\$$ or use $\$$ or using $\$$ or test $\$$ or risk $\$$ or outcome\$) adj4 (reduc $\$$ or declin $\$$ or chang $\$$ or effect $\$$ or increas $\$$ or decreas $\$$ or impact $\$$ or modif $\$$ or lower $\$$ or maintain\$ or maintenance)).ti,ab

79. bleach\$.ti,ab

80. clean\$.ti,ab

81. condom $\$ . t i, a b$

82. contracept $\$ . t i, a b$

83. crack.ti,ab

84. disclos\$.ti,ab

85. incidence.ti,ab

86. inject $\$ . t i, a b$

87. intention\$.ti,ab

88. intercourse.ti,ab

89. needle $\$ . t i, a b$

90. infect $\$$ adj4 (new $\$$ or rate $\$$ or declin $\$$ or reduc $\$$ or prevent $\$$ or lower\$ or decreas\$).ti,ab

91. partner $\$ . t i, a b$

92. seroconver\$.ti, ab

93. sex\$.ti,ab

94. syring $\$ . t i, a b$

95. test $\$ . t i, a b$

96. or $/ 48-95$

97. $\quad 4$ and 47 and 96

Limits: English language, publication types, Date limits 


\section{Appendix C - OVID Search Strategy - EMBASE \\ $\$$ = truncation $\quad *$ focus \\ $\mathrm{ab}=$ abstract $\quad \mathrm{ti}=$ title \\ $/ \mathrm{pc}=$ prevention and control subheading \\ HIV/AIDS/STDs Subject Headings}

$\begin{gathered}\text { 1. } \\ \text { *Human Immunodeficiency } \\ \text { Virus Infection/pc } \\ \text { 2. } \\ \text { *acquired immunodeficiency } \\ \text { syndrome/pc } \\ \text { *sexually transmitted } \\ \text { diseases/pc }\end{gathered}$
or/1-3
Prevention/Intervention Subject Headings
and Keywords

5. Primary Prevention/

6. Preventive Health Service/

7. Health Promotion/

8. Health Care Quality/

9. Patient Care/

10. Randomized Controlled Trials/

11. Evaluation/

12. Contact Examination/

13. Types of Study/

14. Follow Up/

15. Longitudinal Study/

16. Multicenter Study/

17. Randomization/

18. control group.ti,ab

19. control trial.ti, ab

20. controlled trial.ti,ab

21. (rct or rcts).ti,ab

22. case management.ti,ab

23. contact tracing.ti,ab

24. (counseling or counselling).ti,ab

25. (detox or detoxification).ti,ab

26. (drug\$ adj4 treatment\$).ti,ab

27. education\$.ti,ab

28. (effect or effects or effective or effectiveness).ti, ab
29. efficacy.ti,ab

30. evaluation\$.ti, ab

31. impact\$.ti,ab

32. intervention $\$ . t i, a b$

33. needle exchang\$.ti,ab

34. network\$.ti,ab

35. outreach\$.ti,ab

36. partner notification.ti,ab

37. (partner\$ adj4 contact\$ adj4 referral\$).ti, ab

38. (notif\$ adj4 partner\$).ti,ab

39. prevention $\$$.ti, ab

40. program\$.ti,ab

41. random $\$ . t i, a b$

42. rehab\$.ti,ab

43. skill\$.ti,ab

44. syringe exchang $\$$.ti,ab

45. methadone.ti, ab

46. test\$.ti,ab

47. training.ti,ab

48. trial\$.ti,ab

49. $\quad$ or $/ 5-48$

\section{Behavior/Outcomes Subject Headings and Keywords}

50. Behavior/

51. Behavior Therapy/

52. Health Behavior/

53. Infection Risk/

54. High Risk Population/

55. Risk Reduction/

56. Coitus/

57. Abstinence/

58. Sexual Behavior/

59. Safe Sex/

60. Diamorphine/

61. Drug Abuse/

62. Condom/

63. Drug Dependence/

64. Intravenous Drug Abuse/

65. Health Service/

66. "Drug Use"/

67. Substance Abuse/

68. Addiction/

69. Opiate Addiction/

70. Cocaine/ 
71. Cocaine Dependence/

72. heroin.ti,ab

73. cocaine.ti, ab

74. opiate\$.ti,ab

75. opium.ti,ab

76. paraphernalia.ti,ab

77. (treatment\$ adj2 entry).ti,ab

78. (treatment $\$$ adj2 enter\$).ti,ab

79. (abstin\$ or abstain\$).ti,ab

80. drug\$.ti,ab

81. substance.ti,ab

82. (idu or idus or ivdu or ivdus).ti,ab

83. ((behavior\$ or behaviour\$ or activit $\$$ or access $\$$ or utiliz $\$$ or use $\$$ or using $\$$ or test $\$$ or risk $\$$ or outcome\$) adj4 (reduc $\$$ or declin $\$$ or chang $\$$ or effect $\$$ or increas $\$$ or decreas $\$$ or impact $\$$ or modif $\$$ or lower\$ or maintain\$ or maintenance)).ti,ab

84. bleach $\$ . t i, a b$

85. clean\$.ti,ab

86. condom\$.ti,ab

87. contracept\$.ti,ab

88. crack.ti,ab

89. disclos\$.ti,ab

90. incidence.ti,ab

91. inject\$.ti,ab

92. intention\$.ti,ab

93. intercourse.ti,ab

94. needle\$.ti,ab

95. infect $\$$ adj4 (new $\$$ or rate $\$$ or declin $\$$ or reduc $\$$ or prevent $\$$ or lower\$ or decreas $\$$ ).ti,ab

96. partner\$.ti,ab

97. seroconver\$.ti,ab

98. sex\$.ti,ab

99. syring $\$ . t i, a b$

100. test\$.ti,ab

101. or/50-100

102. 4 and 49 and 101

Limits: English language, publication types Date limits 
Appendix D - Journal List for Manual

Search, updated February 2007

Addiction

AIDS

AIDS and Behavior

AIDS Care

AIDS Education and Prevention

AIDS Patient Care and STDs

American Journal of Community Psychology

American Journal of Drug and Alcohol Abuse

American Journal of Preventive Medicine

American Journal of Psychiatry

American Journal of Public Health

Annals of Behavioral Medicine

Archives of Pediatrics \& Adolescent Medicine

Clinical Infectious Diseases

Drug and Alcohol Dependence

Health Education \& Behavior

Health Education Research

Health Psychology

International Journal of STD \& AIDS

JAIDS Journal of Acquired Immune

Deficiency Syndromes

JAMA Journal of the American Medical

Association

Journal of Adolescent Health

Journal of Consulting and Clinical Psychology

Journal of Drug Issues

Journal of Psychoactive Drugs

Journal of Substance Abuse Treatment

Journal of Urban Health

Lancet

Morbidity and Mortality Weekly Report

Psychology of Addictive Behaviors

Public Health Reports

Research in Nursing \& Health

Sexually Transmitted Diseases

Sexually Transmitted Infections

Substance Use \& Misuse 